\title{
Diagnostic Value of Optical Coherence Tomography Image Features for Diagnosis of Basal Cell Carcinoma
}

\author{
Fieke ADAN ${ }^{1,2}$, Klara MOSTERD ${ }^{1,2}$, Nicole W. J. KELLENERS-SMEETS ${ }^{1,2}$ and Patty J. NELEMANS ${ }^{3}$ \\ ${ }^{1}$ Department of Dermatology, Maastricht University Medical Centre, ${ }^{2}$ GROW Research Institute for Oncology and Developmental Biology, \\ Maastricht University and ${ }^{3}$ Department of Epidemiology, Maastricht University, Maastricht, The Netherlands
}

Optical coherence tomography (OCT) is a non-invasive diagnostic method. Numerous morphological OCT features have been described for diagnosis of basal cell carcinoma (BCC). The aim of this study is to evaluate the diagnostic value of established OCT features and to explore whether the use of a small set of OCT features enables accurate discrimination between BCC and non-BCC lesions and between BCC subtypes. For each lesion, the presence or absence of specific OCT features was recorded. Histopathology was used as a gold standard. Diagnostic parameters were calculated for each OCT feature, and multivariate logistic regression analyses were performed to evaluate the loss in discriminative ability when using a small subset of OCT features instead of all features that are characteristic for BCC according to the literature. The results show that the use of a limited number of OCT features allows for good discrimination of superficial BCC from nonsuperficial $B C C$ and non-BCC lesions. The prevalence of BCC was $75.3 \%(225 / 299)$ and the proposed diagnostic algorithm enabled detection of $97.8 \%$ of BCC lesions $(220 / 225)$. Subtyping without the need for biopsy was possible in 132 of 299 patients (44\%), with a predictive value for presence of superficial BCC of $\mathbf{8 4 . 3} \%$ vs $\mathbf{9 8 . 8} \%$ for presence of non-superficial BCC.

Key words: basal cell carcinoma; optical coherence tomography; diagnostic; non-invasive.

Accepted Nov 1, 2021; Epub ahead of print Nov 1, 2021

Acta Derm Venereol 2021; 101: adv00607.

Corr: Fieke Adan, Department of Dermatology, Maastricht University Medical Centre, P. Debyelaan 25, NL-6229 HX Maastricht, The Netherlands. E-mail: fieke.adan@mumc.nl

$\mathrm{T}$ he incidence of non-melanoma skin cancer (NMSC) has increased over the past decades, with basal cell carcinoma (BCC) being the most prevalent cancer diagnosed in the Caucasian population worldwide (1-4). Although there are many histopathological subtypes, a simplified classification by Rippey roughly groups all BCCs into 3 subtypes: superficial, nodular, and aggressive (5). Currently, histopathological examination of a punch biopsy is the gold standard to discriminate BCC from alternative diagnoses and to determine the histopathological subtype (6).

In recent years, optical coherence tomography (OCT) has emerged as a promising non-invasive diagnostic method for diagnosis of BCC, generating real-time in

\section{SIGNIFICANCE}

Optical coherence tomography has emerged as a promising non-invasive diagnostic method for diagnosis of basal cell carcinoma. Numerous morphological optical coherence tomography features have been described for diagnosis and subtyping of basal cell carcinoma, but it is not known which features are most discriminative. Therefore, the aim of this study is to evaluate the diagnostic value of established optical coherence tomography image features for diagnosis and subtyping of basal cell carcinoma. Secondly, the study explored whether the use of a small set of optical coherence tomography features with the highest predictive value enables accurate discrimination between basal cell carcinoma and non-basal cell carcinoma lesions and between basal cell carcinoma subtypes, which might be of benefit for novice optical coherence tomography assessors commencing optical coherence tomography training.

vivo cross-sectional images of tissue microarchitecture with a depth of approximately $1.5 \mathrm{~mm}$ (7). OCT is based on light interferometry; the interference of 2 optical beams reflected by tissue produces different shades in the black and white spectrum. Despite the high lateral resolution of $<7.5 \mathrm{~mm}$ and axial resolution of $<5 \mathrm{~mm}$, imaging of individual cells is not possible. OCT is therefore suitable for pattern recognition in tissue, similar to, for example, ultrasound, allowing for the identification of morphological features of $\mathrm{BCC}$, which have been established in recent years (8-12). In 2015, Hussain et al. systematically reviewed diagnostic criteria for BCC. The authors evaluated 17 studies and found that, in $100 \%$ of these studies, rounded dark (hyporeflective) structures in the upper dermis, surrounded by a bright (hyper-reflective) halo, sometimes surrounded by a hyporeflective border and disruption of epidermal layering, were described as characteristic for BCC (8). The rounded dark structures resemble the basaloid cell nests seen in histology, the hyper-reflective halo surrounding the rounded structures corresponds to the surrounding tumour stroma and a hyporeflective border at the periphery resembles the peripheral palisading at the margins of basaloid cell nests (13). Other features that have been described for BCC are shown in Table I, some of which are illustrated in Fig. 1 (9-12).

It is not known which OCT features are most discriminative for BCC diagnosis. Therefore, the primary aim of 
Table I. Optical coherence tomography (OCT) image features and corresponding histopathology features (8-12, 19)

\begin{tabular}{ll}
\hline OCT & Histopathology \\
\hline Epidermis & \\
Superficial scaling/crust/ulceration & $\begin{array}{l}\text { Superficial scaling/crust/ulceration } \\
\text { Atrophy of the epidermis } \\
\text { Atrophy of the epidermis }\end{array}$ \\
$\begin{array}{l}\text { Thickening of the epidermis } \\
\text { Shickeng of the epidermis }\end{array}$ & Superficial basaloid nests with a firm connection to the epidermis \\
Protrusions into the upper dermis with dark rim & Interrupted/poorly defined \\
Dermo-epidermal junction & \\
Interrupted/poorly defined & Basaloid nests in the dermis \\
Dermis & Basaloid nests with necrotic cell debris in centre \\
Signal-poor ovoid structures/rounded dark structures & Peripheral palisading \\
Ovoid structures with bright centre & Collagen compression between adjacent nests \\
Dark rim/hyporeflective border & Dilated capillaries \\
Bright (hyper-reflective) peritumoural stroma/hyper-reflective halo & Nests in morpheaform and micronodular basal cell carcinoma, respectively \\
Prominent vessels & Area of liquefactive necrosis \\
Small ovoid signal-poor structures "Shoal of fish"' or "Bunch of grapes" & \\
Dark/black (areflective) areas/cysts &
\end{tabular}

this study is to evaluate the diagnostic value of established morphological OCT features that can be used for diagnosis and subtyping of $\mathrm{BCC}$. The second objective is to explore whether the use of a small set of features with the highest predictive value enables accurate discrimination between $\mathrm{BCC}$ and non-BCC lesions and between BCC subtypes.

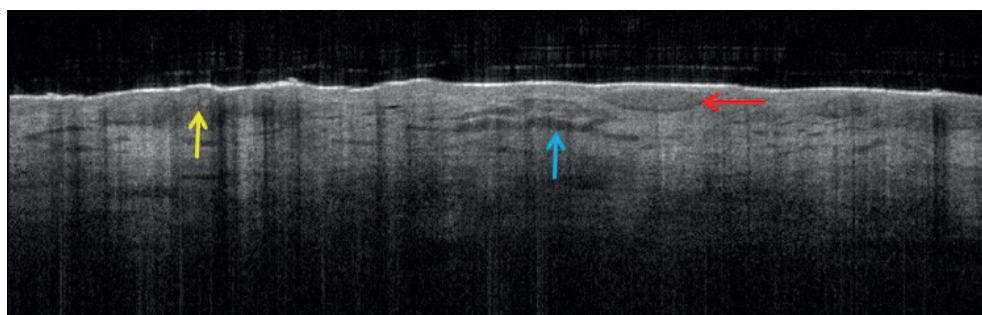

A

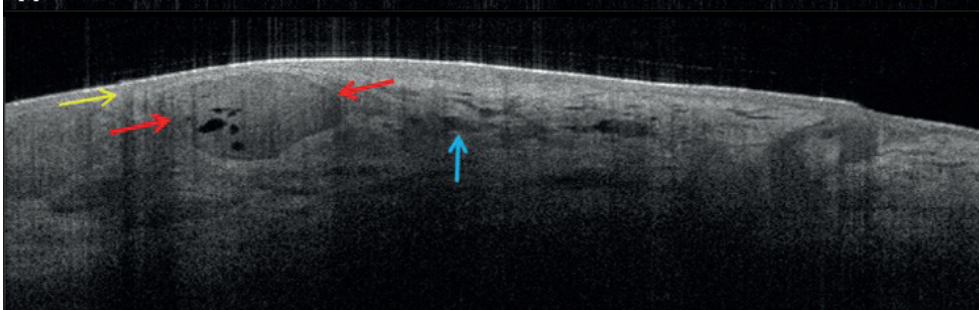

B

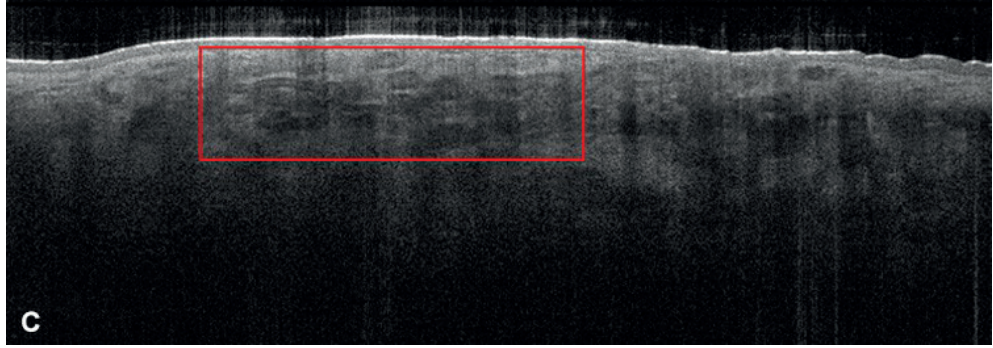

Fig. 1. Optical coherence tomography (OCT) images of different basal cell carcinoma (BCC) subtypes. (A) OCT image of a superficial BCC. Red arrow points towards a protrusion into the upper dermis with a dark rim, corresponding to a basaloid cell nest with a firm connection to the epidermis (10). Vessels in the upper dermis are dilated (blue arrow) and the dermal epidermal junction (DEJ) is disrupted (yellow arrow). (B) OCT image of a nodular BCC. Red arrows point towards a fully encompassing signal-poor ovoid structure located in the dermis with a dark rim and bright peritumoural stroma. Inside the nest, well-circumscribed black areas are observed, representing signs of liquefactive necrosis (19). Vessels in the upper dermis are dilated and directed towards the tumour nests (blue arrow). The epidermis above the nest is atrophic, the DEJ is disrupted (yellow arrow). (C) OCT image of an aggressive BCC. Inside the red rectangle, smaller signal-poor ovoid structures with surrounding bright peritumoural stroma are visible, also described as "shoal of fish" or "bunch of grapes", indicating an aggressive BCC subtype (9).

\section{MATERIALS AND METHODS}

Data for this study were derived from 1 arm of a randomized multi-centre non-inferiority trial in 1 academic and 2 general Dutch hospitals: the dermatology outpatient clinic of Maastricht University Medical Centre+ (Maastricht), Catharina Hospital (Eindhoven) and Zuyderland Medical Centre (Heerlen). Included were consecutive patients (18 years or older) with an indication for skin biopsy of a lesion clinically suspected for BCC. Excluded were patients with a lesion located in the "H-zone" of the face, patients with a large BCC referred to our head and neck tumour working group, and patients who were unable to sign informed consent. The marked biopsy area of the clinically most aggressive part was scanned with OCT without any preparation of the skin in advance (Vivosight Multi-beam Swept-Source Frequency Domain OCT, Michelson Diagnostics, Maidstone, Kent, UK).

OCT scanning and interpretations were conducted by a single experienced investigator (FA), who used morphological OCT features that are characteristic for identifying BCC lesions (8-10, 14). For each lesion, the presence or absence of specific morphological OCT features was recorded (Table I). Features relating to the epidermis included: superficial scaling/ crust/ulceration, protrusions into the dermis with a dark rim, atrophy or thickening of epidermis, dermal epidermal junction (DEJ) interrupted/poorly defined. Features relating to the dermis included: signal-poor ovoid structures, ovoid structures with bright centre, dark rim, bright peritumoural stroma, prominent vessels, black areas or cysts and small signal-poor ovoid structures ("shoal of fish" or "'bunch of grapes" appearance).

The histopathological result from a punch biopsy or excision biopsy was used as a gold standard. Histopathological examination was performed by a specialized dermato-pathologist, who was blinded to the OCT diagnosis. BCC subtypes were classified as superficial, nodular, or aggressive BCC. 
The study was approved by the Medical Ethical Committee of MUMC+(METC 18-043). All patients provided written informed consent.

\section{Statistical analysis}

The distribution of baseline characteristics in patients who underwent OCT examination was summarized by absolute numbers and percentages for categorical variables and by mean values with standard deviations (SD) or median with range for continuous variables. Diagnostic parameters were calculated for each morphological OCT feature. Diagnostic odds ratios (DOR) with $95 \%$ confidence intervals $(95 \% \mathrm{CI})$ were calculated using univariate logistic regression analyses.

Subsequently, multivariate logistic regression analyses were performed to evaluate the loss in discriminative ability when using a small subset of morphological features instead of all morphological features that are characteristic for BCC according to the literature (8-10). Histologically verified presence or absence of BCC was the dependent variable. Discriminative ability was expressed as the area under the receiver operating characteristic curve (AUC) and differences in AUCs between models were tested for significance using an algorithm for paired comparison of AUCs from DeLong et al. (15).

Similar analyses were performed to identify subsets of features that can be used for accurate subtyping. These analyses were restricted to the subgroup of patients with histologically confirmed BCC. Discrimination between superficial BCC and non-superficial BCC subtypes was considered most relevant for clinical practice, since superficial BCC can be treated non-invasively. Therefore, histologically verified superficial BCC vs non-superficial BCC subtypes was used as dependent variable. A DOR $>1$ indicates that the presence of a feature is indicative for the presence of superficial BCC (sBCC). $p$-values $\leq 0.05$ were considered to indicate statistical significance. Analyses were performed using IBM SPSS Statistics version 24 and Stata version 14.

\section{RESULTS}

A total of 598 patients with 598 skin lesions clinically suspicious for BCC were included in the randomized trial between March 2019 and September 2020. Data for 299 patients, who were randomized to the OCT group, were used for this study. According to histological diagnosis,
Table II. Baseline characteristics of 299 patients who underwent optical coherence tomography (OCT) examination

\begin{tabular}{ll}
\hline Characteristic & \\
\hline Age, years, median (SD) & $72(21-95)$ \\
Sex, $n$ (\%) & \\
Male & $164(54.8)$ \\
Female & $135(45.2)$ \\
Localization, $n$ (\%) & \\
Head/neck & $94(31.4)$ \\
Upper anterior chest & $37(12.4)$ \\
Trunk & $89(29.8)$ \\
Extremities & $79(26.4)$ \\
Histological diagnoses, $n$ (\%) & \\
BCC & $225(75.3)$ \\
No BCC & $74(24.7)$ \\
BCC subtypes, $n$ (\%) & \\
Superficial & $66(29.3)$ \\
Nodular & $79(35.1)$ \\
Aggressive (morpheaform/micronodular) & $12(5.3)$ \\
Mixed & $68(30.2)$ \\
Other diagnoses (non-BCC), $n$ (\%) & \\
Benigna & $34(11.4)$ \\
Actinic keratosis & $24(8.0)$ \\
Bowen's disease & $9(3.0)$ \\
SCC & $5(1.7)$ \\
Superficial spreading malignant melanoma & $1(0.3)$ \\
Atypical fibroxanthoma & $1(0.3)$ \\
\hline
\end{tabular}

ancluding: sebaceous gland hyperplasia and/or adenoma, dermatofibroma, derma naevus, seborrhoeic keratosis, scar, benign lichenoid keratosis, acute folliculitis, neurofibroma, trichofolliculoma, venous stasis dermatitis, sclerosing dermatitis, excoriation, dilated hair follicle, angioma, chronic inflammation, eczema, apocrine hidrocystoma, epidermoid cyst.

BCC: basal cell carcinoma; SCC: squamous cell carcinoma.

$225(75.3 \%)$ patients had a BCC and 74 had an alternative diagnosis. Of these $225 \mathrm{BCCs}, 66$ were superficial, 79 nodular and 12 aggressive. The remaining $68 \mathrm{BCCs}$ were of a mixed subtype (Table II).

Discriminating between basal cell carcinoma and nonbasal cell carcinoma

The diagnostic parameters for each morphological OCT feature described as relevant for discrimination between BCC and non-BCC lesions are shown in Table III. A dark rim is the strongest positive predictor of $\mathrm{BCC}$ (odds ratio (OR) 64.11, 95\% CI 27.02-152.11), followed by bright

Table III. Diagnostic parameters for discrimination between basal cell carcinoma (BCC) and non-BCC. Odds ratios (OR) of univariate logistic regression analyses and $p$-values

\begin{tabular}{|c|c|c|c|c|c|c|}
\hline Characteristic & $\begin{array}{l}\text { Presence in } \\
\text { BCC }(\%)^{*} \\
\text { (Sensitivity) }\end{array}$ & $\begin{array}{l}\text { Absence in } \\
\text { non-BCC (\%) } \\
\text { (Specificity) }\end{array}$ & $\begin{array}{l}\text { Probability of } \\
\text { BCC if present } \\
\text { (PPV) }\end{array}$ & $\begin{array}{l}\text { Probability of } \\
\text { non-BCC if } \\
\text { absent (NPV) }\end{array}$ & Odds ratio $(95 \% \mathrm{CI})$ & $\begin{array}{l}p- \\
\text { value }\end{array}$ \\
\hline \multicolumn{7}{|l|}{ Epidermis } \\
\hline Superficial scaling/crust/ulceration & $57.3(129 / 225)$ & $40.5(30 / 74)$ & $74.6(129 / 173)$ & $23.8(30 / 126)$ & $0.92(0.54-1.56)$ & 0.79 \\
\hline Atrophy of the epidermis & $40.9(92 / 225)$ & $94.6(70 / 74)$ & $95.8(92 / 96)$ & $34.5(70 / 203)$ & $12.11(4.27-34.32)$ & $<0.0001$ \\
\hline Thickening of the epidermis & $16.0(36 / 226)$ & $31.1(23 / 74)$ & $41.4(36 / 87)$ & $10.8(23 / 212)$ & $0.09(0.05-0.16)$ & $<0.0001$ \\
\hline Protrusions into upper dermis with dark rim & $61.3(138 / 225)$ & $90.5(67 / 74)$ & $95.2(138 / 145)$ & $43.5(67 / 154)$ & $15.18(6.66-34.59)$ & $<0.0001$ \\
\hline \multicolumn{7}{|l|}{ Dermal-epidermal junction } \\
\hline $\begin{array}{l}\text { Dermal-epidermal junction poorly defined/interrupted } \\
\text { Dermis }\end{array}$ & $74.7(168 / 225)$ & $75.7(56 / 74)$ & $90.3(168 / 186)$ & $49.6(56 / 113)$ & $9.17(4.98-16.88)$ & $<0.0001$ \\
\hline Signal-poor ovoid structures & $69.8(157 / 225)$ & $79.7(59 / 74)$ & $91.3(157 / 172)$ & $46.5(59 / 127)$ & $9.08(4.82-17.12)$ & $<0.0001$ \\
\hline Ovoid structures with bright centre & $28.9(65 / 225)$ & $93.2(69 / 74)$ & $92.9(65 / 70)$ & $30.1(69 / 229)$ & $5.61(2.16-14.53)$ & $<0.0001$ \\
\hline Dark rim & $96.4(217 / 225)$ & $70.3(52 / 74)$ & $90.8(217 / 239)$ & $86.7(52 / 60)$ & $64.11(27.02-152.11)$ & $<0.0001$ \\
\hline Bright peritumoural stroma & $81.3(183 / 225)$ & $91.9(68 / 74)$ & $96.8(183 / 189)$ & $61.8(68 / 110)$ & $49.38(20.09-121.40)$ & $<0.0001$ \\
\hline Prominent vessels & $56.9(128 / 225)$ & $44.6(33 / 74)$ & $75.7(128 / 169)$ & $25.4(33 / 130)$ & $1.06(0.63-1.80)$ & 0.89 \\
\hline Shoal of fish/bunch of grapes & $22.2(50 / 225)$ & $97.3(72 / 74)$ & $96.2(50 / 52)$ & $29.1(72 / 247)$ & $10.29(2.44-43.40)$ & $<0.0001$ \\
\hline Black areas, cysts & $27.6(62 / 225)$ & $95.9(71 / 74)$ & $95.4(62 / 65)$ & $30.3(71 / 234)$ & $9.00(2.73-29.64)$ & $<0.0001$ \\
\hline
\end{tabular}

*Data subset includes 225 BCCs and 74 non-BCCs.

PPV: positive predictive value; NPV: negative predictive value; $95 \%$ CI: $95 \%$ confidence interval. 
peritumoural stroma (OR 49.38, 95\% CI 20.09-121.40). Protrusions into the upper dermis with a dark rim (OR $15.18,95 \%$ CI $6.66-34.59)$, poorly defined/interrupted dermal epidermal junction (DEJ) (OR 9.17, 95\% CI 4.98-16.88) and signal-poor ovoid structures (OR 9.08, $95 \%$ CI $4.82-17.12$ ) were also strongly associated with presence of BCC. The ORs were statistically significant $(p<0.0001)$ for all features, except superficial scaling/crust/ulceration $(p=0.79)$ and prominent vessels $(p=0.89)$.

The AUC of the full multivariate logistic model including all 12 morphological features was $95.2 \%$ (95\% CI 91.8-98.7\%), The AUC of a model including the 5 features with the highest DORs was $94.7 \%$ (95\% CI 91.4-98.1\%). A final model included the 4 features that were most discriminative, based on clinical experience of the investigator: signal-poor ovoid structures, dark rim, bright peritumoural stroma and protrusions into the upper dermis with a dark rim. For this model, the AUC decreased to $94.1 \%$ (95\% CI 90.8-97.3\%). However, the decreases in AUC when using 4 or 5 features instead of all features were minor and non-significant ( $p=0.49$ and $p=0.24$, respectively). Based on these results we conclude that 4 features can be used to discriminate between $\mathrm{BCC}$ and non-BCC without significant loss of diagnostic performance.

\section{Discriminating between superficial basal cell carcinoma and non-superficial basal cell carcinoma subtypes}

Table IV shows the frequency of each morphological feature, as well as diagnostic parameters for discrimination between superficial BCC and non-superficial BCC subtypes. The diagnostic odds ratio (DOR) was significant for 8 features. The high DOR for "protrusions into the upper dermis with a dark rim" and an "interrupted DEJ" indicates that the presence of these features is strong- ly indicative for presence of superficial BCC (sBCC). The other 6 features: $(i)$ atrophy of the epidermis, (ii) signal-poor ovoid structures, (iii) ovoid structures with bright centre, (iv) bright peritumoural stroma, $(v)$ shoal of fish/bunch of grapes, and (vi) black areas, cysts are associated with DORs that are significantly lower than 1 , indicating that these features are highly predictive for non-superficial BCC.

The AUC of the full multivariate logistic model including all 12 features was 95.5\% (95\% CI 93.0-98.0). The use of a model including the 8 features that were associated with a significantly increased or decreased DOR resulted in a comparable AUC $(p=0.88)$ of $95.6 \%(95 \%$ CI 93.2-98.0\%). Use of a subset of 6 of these 8 features (excluding "bright peritumoural stroma" and "interrupted DEJ") led to a significant ( $p=0.01)$ decrease in the AUC (94.0\% with 95\% CI 91.1-97.0\%).

\section{Diagnostic algorithm}

Fig. 2 proposes a diagnostic algorithm for diagnosis and subtyping of BCC. First, to discriminate between BCC and non-BCC lesions, one can use the 4 features: ( $i$ ) dark rim, (ii) bright peritumoural stroma, (iii) protrusions, and (iv) signal-poor ovoid structures. If 1 or more of these features are present, the probability of BCC is $89.4 \%$ (PPV), whereas if all 4 features are absent the probability of a non-BCC lesion is $90.6 \%$ (NPV).

With respect to subtyping, absence of the feature "protrusions into the upper dermis with a dark rim" indicates a high probability of non-superficial BCC. However, when this feature is present, both superficial and non-superficial $\mathrm{BCC}$ can be present. For further discrimination, additional features that increase the probability of non-superficial BCC can be used: (i) atrophy of the epidermis, (ii) signal-poor ovoid structures, (iii) ovoid structures with bright centre, (iv) shoal of fish/

Table IV. Prevalence of morphological features in superficial basal cell carcinoma (BCC) and non-superficial BCC subtypes in patients with histologically verified BCC. Odds ratios (OR) of univariate logistic regression analyses and $p$-values

\begin{tabular}{|c|c|c|c|c|c|c|}
\hline Characteristic & $\begin{array}{l}\text { Presence in } \\
\text { sBCC (\%) } \\
n=66 \\
\text { (Sensitivity) }\end{array}$ & $\begin{array}{l}\text { Absence in non- } \\
\text { superficial BCC } \\
\text { subtype (\%) } \\
n=159 \text { (Specificity) }\end{array}$ & $\begin{array}{l}\text { Probability of } \\
\text { SBCC if present } \\
\text { (PPV) }\end{array}$ & $\begin{array}{l}\text { Probability of non- } \\
\text { superficial BCC } \\
\text { subtype if absent } \\
\text { (NPV) }\end{array}$ & $\begin{array}{l}\text { Odds ratio* }(95 \% \\
\text { CI) }\end{array}$ & $\begin{array}{l}p- \\
\text { value }\end{array}$ \\
\hline \multicolumn{7}{|l|}{ Epidermis } \\
\hline Superficial scaling/crust/ulceration & $56.1(37 / 66)$ & $42.1(67 / 159)$ & $28.7(37 / 129)$ & $69.8(67 / 96)$ & $0.93(0.52-1.66)$ & 0.88 \\
\hline Atrophy of the epidermis & $9.1(6 / 66)$ & $45.9(73 / 159)$ & $6.5(6 / 92)$ & $54.9(73 / 133)$ & $0.09(0.04-0.21)$ & $<0.001$ \\
\hline Thickening of the epidermis & $22.7(15 / 66)$ & $86.8(138 / 159)$ & $41.7(15 / 36)$ & $73.0(138 / 189)$ & $1.93(0.93-4.04)$ & 0.11 \\
\hline Protrusions into upper dermis with dark rim & $95.5(63 / 66)$ & $52.8(84 / 159)$ & $45.7(63 / 138)$ & $96.6(84 / 87)$ & $23.52(7.09-78.04)$ & $<0.001$ \\
\hline \multicolumn{7}{|l|}{ Dermal-epidermal junction } \\
\hline Dermal-epidermal junction poorly defined & $98.5(65 / 66)$ & $35.2(56 / 159)$ & $38.7(65 / 168)$ & $98.2(56 / 57)$ & $35.34(4.78-261.55)$ & $<0.001$ \\
\hline \multicolumn{7}{|l|}{ Dermis } \\
\hline Signal-poor ovoid structures & $19.7(13 / 66)$ & $9.4(15 / 159)$ & $8.3(13 / 157)$ & $22.1(15 / 68)$ & $0.03(0.01-0.06)$ & $<0.001$ \\
\hline Ovoid structures with bright centre & $9.1(6 / 66)$ & $62.9(100 / 159)$ & $9.2(6 / 65)$ & $62.5(100 / 160)$ & $0.17(0.07-0.42)$ & $<0.001$ \\
\hline Dark rim & $95.5(63 / 66)$ & $3.1(5 / 159)$ & $29.0(63 / 217)$ & $62.5(5 / 8)$ & $0.68(0.16-2.94)$ & 0.70 \\
\hline Bright peritumoural stroma & $65.2(43 / 66)$ & $11.9(19 / 159)$ & $23.5(43 / 183)$ & $45.2(19 / 42)$ & $0.25(0.13-0.51)$ & $<0.001$ \\
\hline Prominent vessels & $43.9(29 / 66)$ & $37.7(60 / 159)$ & $22.7(29 / 128)$ & $61.9(60 / 97)$ & $0.48(0.27-0.85)$ & 0.01 \\
\hline Shoal of fish/bunch of grapes & $0.0(0 / 66)$ & $68.6(109 / 159)$ & $0.0(0 / 50)$ & $62.3(109 / 175)$ & $0^{a}$ & $<0.001$ \\
\hline Black areas, cysts & $9.1(6 / 66)$ & $64.8(103 / 159)$ & $9.7(6 / 62)$ & $63.2(103 / 163)$ & $0.18(0.08-0.45)$ & $<0.001$ \\
\hline
\end{tabular}

${ }^{\mathrm{a} C}$ Cannot be calculated.

*An odds ratio $>1$ indicates higher probability of superficial BCC and an odds ratio $<1$ indicates higher probability of non-superficial subtype.

PPV: positive predictive value; NPV: negative predictive value; $95 \%$ CI: $95 \%$ confidence interval. 


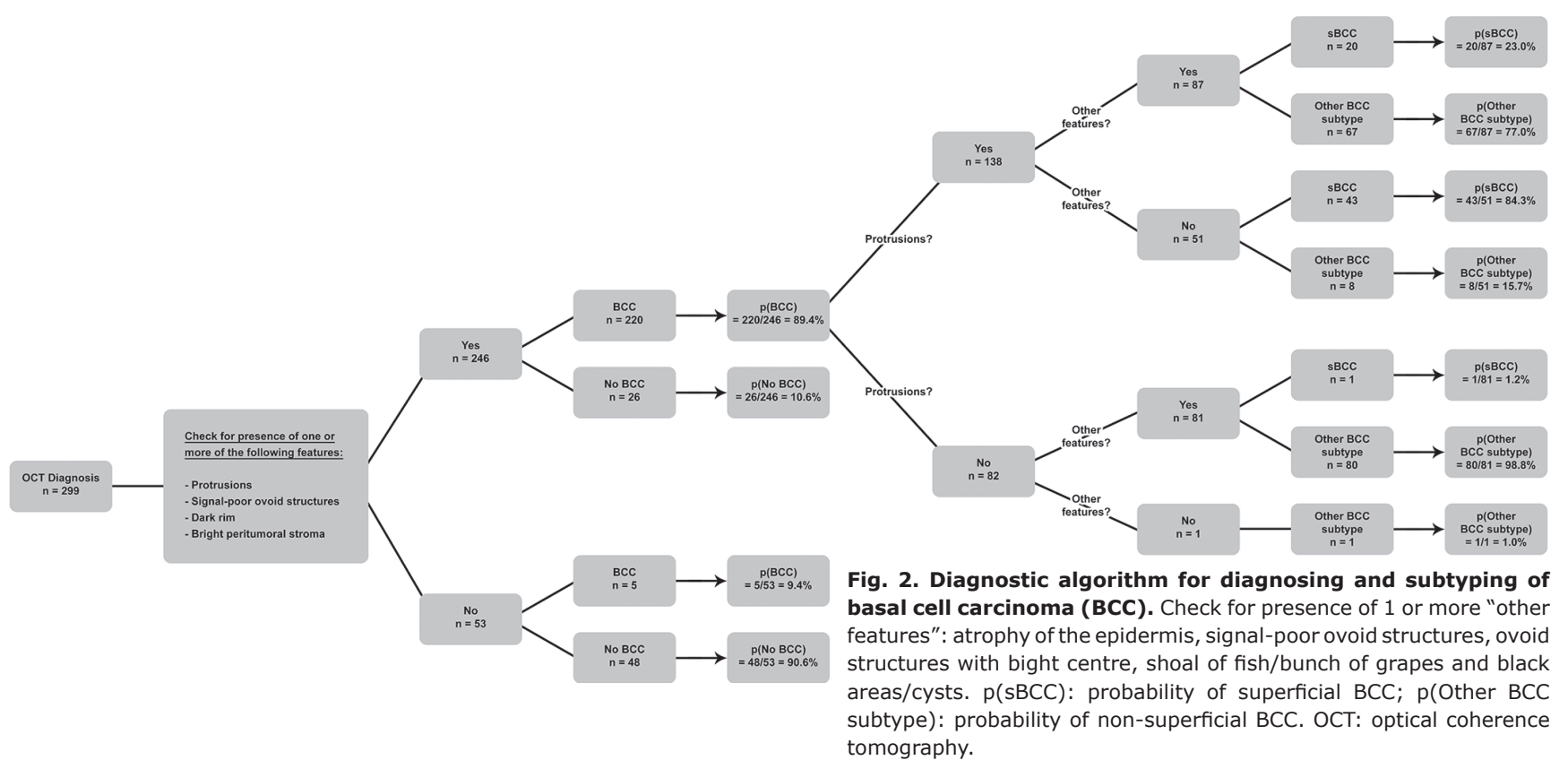

bunch of grapes, or $(v)$ black areas, cysts. If all 5 features are absent and protrusions are present $(n=51)$, the probability of superficial BCC is $84.3 \%$. If 1 or more of the 5 features are present and protrusions are absent $(n=81)$, there is a high probability of non-superficial BCC subtype $(98.8 \%)$. In case of other combinations, there remains too much doubt and a punch biopsy is still necessary to determine the histological subtype. For instance, when both protrusions and 1 or more of the other 5 features are present, the probability of superficial BCC and nonsuperficial BCC is $23 \%$ and $77 \%$, respectively.

Summarizing, the use of the diagnostic algorithm enabled detection of $97.8 \%$ of BCC lesions (220/225). In 132 of 299 patients (44\%) a diagnosis of subtype could be made with high predictive value for presence of superficial BCC $(84.3 \%)$ vs presence of non-superficial BCC $(98.8 \%)$ without the need for biopsy.

\section{DISCUSSION}

This study evaluated the diagnostic value of morphological OCT features for BCC diagnosis. With the features: (i) dark rim, (ii) bright peritumoural stroma, (iii) protrusions into the upper dermis with a dark rim, and (iv) signal-poor ovoid structures (all with PPV $>90 \%$ ) a good discrimination between $\mathrm{BCC}$ and non-BCC lesions is possible. With regard to subtyping, "protrusions into the upper dermis with a dark rim" are visible in the vast majority of superficial BCCs and absence of this feature is highly predictive of non-superficial BCC when other BCC features are present. However, if "protrusions" are present, a conclusive diagnosis cannot be made. In this situation, 5 other features that are highly predictive of non-superficial BCC have to be used.
Accurate diagnosis of $\mathrm{BCC}$ with $\mathrm{OCT}$, or other noninvasive methods, would enable the use of a one-stop shop approach in patients with a lesion clinically suspected for BCC. This approach requires that superficial BCCs can be distinguished from non-superficial BCCs in a substantial proportion of patients. Patients with superficial BCC can be treated non-invasively (i.e. imiquimod cream, photodynamic therapy). Therefore, these patients would benefit most from accurate non-invasive diagnosis.

In case of high suspicion of $\mathrm{sBCC}$, non-invasive treatment can be discussed with the patient and initiated in the same visit, and an invasive procedure with risk of pain, bleeding and scar formation can be prevented. In case of high suspicion of non-superficial BCC, a surgical excision can be planned immediately. This one-stop shop approach could result in a reduction in the number of biopsies and is expected to be more efficient, patient friendly and cost-effective than regular care, where diagnosis and treatment is based on the histopathological result of a punch biopsy $(16,17)$.

The proposed diagnostic algorithm offers a systematic approach towards discrimination of superficial BCC from non-superficial BCC and non-BCC lesions using OCT. The first step, discrimination of BCC from non-BCC lesions, is possible with 4 features (PPV of 89.4) There is a risk (of approximately $10 \%$ ) that a lesion that is diagnosed as BCC by OCT is not a BCC. The prevention of misclassification of another cutaneous malignancy as $\mathrm{BCC}$ is the biggest challenge. Cheng et al. evaluated the diagnostic accuracy of OCT in lesions for which sBCC was considered in the differential diagnosis. In their series, one amelanotic melanoma was misclassified as sBCC. In the current study cohort, one patient had a 
superficial spreading malignant melanoma, clinically highly suspected for BCC. However, upon OCT examination, no $\mathrm{BCC}$ features were present, so this case was diagnosed as a non-BCC lesion with an indication for biopsy. The risk of misclassification of an amelanotic melanoma as BCC is small, but future research on morphological OCT features that can help to distinguish melanoma from BCC is needed.

The proposed diagnostic algorithm also enables discrimination of sBCC from non-superficial BCCs and non-BCC lesions, but there remains a $15.7 \%$ chance that non-superficial BCC is misclassified as $\mathrm{SBCC}$, if protrusions are present and 1 or more of the other 5 features are absent. Such misclassifications may result in a higher risk of residual or recurrent $\mathrm{BCC}$ if non-invasive treatment is chosen. To verify that misclassifications by OCT do not compromise patient safety, a randomized controlled trial, which compares effectiveness of an OCT-guided diagnosis with regular care (biopsy) is necessary.

The diagnostic algorithm uses 6 features for subtyping, although the discriminative ability of a multivariate diagnostic model using 6 features was significantly worse than that of a model with 8 features, which also included "bright peritumoural stroma" and "interrupted DEJ". Still, the latter 2 features were not used in the algorithm. The reason is that the multivariate model evaluates the probability of sBCC given all combinations of the presence and/or absence of features, whereas the diagnostic algorithm considers only a limited number of possible combinations. Absence of "protrusions" in combination with 1 or more of features indicative of non-superficial $\mathrm{BCC}$ results in high probability of non-superficial BCC being present. However, in this respect, addition of the feature "bright peritumoural stroma", which is indicative of non-superficial BCC, was not helpful, because its prevalence is also high in superficial BCC. The feature "interrupted DEJ" was also left out. It had little added value, because it nearly always occurred in combination with the feature "protrusions into the upper dermis with a dark rim" (except for 2 lesions).

In this study, the selection of features that are useful for diagnosis and subtyping of $\mathrm{BCC}$ was based on reports from the literature. A limitation is that there is high variability in the use of terminology in the literature. Describing the 4 features that discriminate well between BCC and non-BCC lesions, Hussain et al. (8) referred to: $(i)$ rounded dark structures in the upper dermis (corresponding to signal-poor ovoid structures); (ii) surrounded by a hyper-reflective halo (bright peritumoural stroma); (iii) possibly surrounded by a hyporeflective border (dark rim); and (iv) disruption of epidermal layering (poorly defined/interrupted DEJ). Cheng et al. (10) concluded that "protrusions into the upper dermis with a dark rim" are highly predictive for superficial BCC, and described this feature as "hyporeflective ovoid structures with firm attachment to the DEJ and a clefting region focused or solely visible at the inferior margin". The high prevalence of an atrophic epidermis and ovoid structures with bright centre (referred to as intranodular small bright dots) in nodular BCC has also been reported by other studies $(11,12)$. Since the evidence for the use of OCT for BCC diagnosis is increasing, more uniformity in terminology is desirable for future implementation of OCT for BCC diagnosis. This study used conventional OCT, whereas the use of dynamic OCT provides additional information by visualizing the vascular patterns and thus allows for better differentiation between BCC subtypes. Therefore, with use of dynamic OCT the proposed diagnostic algorithm may lead to even better diagnostic performance, which can be evaluated in future studies (18).

A limitation of this study is that predictive values, such as PPV and NPV, are highly dependent on the prevalence of BCC and SBCC subtypes in a study population. In this study, patients were included with lesions suspected for BCC, based on clinical and dermoscopic examination by a dermatologist, leading to a study population with a high prevalence of BCC (75.3\%). In other study populations, where for BCC suspected lesions would have been selected by physicians with less experience in clinical and dermoscopic examination, the prevalence of $\mathrm{BCC}$ might be lower, which could result in different predictive values of the morphological features. Furthermore, the proposed diagnostic algorithm is intended as support for OCT users who do not yet have much experience with interpretation of OCT images. The trial aimed to evaluate whether OCT-guided diagnosis and treatment does not compromise patient safety compared with regular care (always biopsy). For ethical reasons, it was decided to not yet include high-risk patients with large lesions or lesions in the "H-zone". Furthermore, the H-zone surface areas are often convex or concave, making it more difficult to obtain an OCT image of sufficient quality. More studies are therefore required to determine whether OCT is suitable in this subpopulation. Also, the diagnostic algorithm needs to be validated in future studies.

In conclusion, the results of this study show that the use of a limited number of morphological features allows for good discrimination of superficial BCC from non-superficial BCC and non-BCC lesions, with the potential to obviate the need for punch biopsy in $44 \%$ of patients. Hence, novice OCT assessors commencing OCT training could focus initially on recognizing these selected features.

\section{ACKNOWLEDGEMENTS}

The study was financed by a grant from the Netherlands Organisation for Health Research and Development (ZonMw; 852001928). ZonMw is a governmental institution financing research to improve healthcare in the Netherlands. The funding source had no role in the design or conduct of the study; collection, management, analysis or interpretation of the data; or preparation, review or approval of the manuscript. 
The authors have no conflicts of interest to declare.

\section{REFERENCES}

1. Lomas A, Leonardi-Bee J, Bath-Hextall F. A systematic review of worldwide incidence of nonmelanoma skin cancer. $\mathrm{Br}$ J Dermatol 2012; 166: 1069-1080.

2. Flohil SC, de Vries E, Neumann HA, Coebergh JW, Nijsten $\mathrm{T}$. Incidence, prevalence and future trends of primary basal cell carcinoma in the Netherlands. Acta Derm Venereol 2011; 91: 24-30.

3. Flohil SC, Seubring I, van Rossum MM, Coebergh JW, de Vries E, Nijsten T. Trends in Basal cell carcinoma incidence rates: a 37-year Dutch observational study. Invest Dermatol 2013; 133: 913-918.

4. Rogers HW, Weinstock MA, Feldman SR, Coldiron BM. Incidence estimate of nonmelanoma skin cancer (keratinocyte carcinomas) in the U.S. population, 2012. JAMA Dermatol 2015; 151: 1081-1086.

5. Rippey J]. Why classify basal cell carcinomas? Histopathology 1998; 32: 393-398.

6. NVDV. Multi-disciplinary evidence-based guideline basal cel carcinoma. Available from: www.oncoline.nl. Utrecht, 2015.

7. Cheng HM, Guitera P. Systematic review of optical coherence tomography usage in the diagnosis and management of basal cell carcinoma. Br J Dermatol 2015; 173: 1371-1380.

8. Hussain AA, Themstrup L, Jemec GBE. Optical coherence tomography in the diagnosis of basal cell carcinoma. Arch Dermatol Res 2015; 307: 1-10.

9. Ulrich $M$, von Braunmuehl $T$, Kurzen $H$, Dirschka $T$, Kellner C, Sattler E, et al. The sensitivity and specificity of optical coherence tomography for the assisted diagnosis of nonpigmented basal cell carcinoma: an observational study. $\mathrm{Br}$ J Dermatol 2015; 173: 428-435.

10. Cheng HM, Lo S, Scolyer R, Meekings A, Carlos G, Guitera P. Accuracy of optical coherence tomography for the diagnosis of superficial basal cell carcinoma: a prospective, consecu- tive, cohort study of 168 cases. Br J Dermatol 2016; 175: $1290-1300$.

11. von Braunmuhl T, Hartmann D, Tietze JK, Cekovic D, Kunte C, Ruzicka T, et al. Morphologic features of basal cell carcinoma using the en-face mode in frequency domain optical coherence tomography. J Eur Acad Dermatol Venereol 2016; 30: 1919-1925.

12. Wahrlich C, Alawi SA, Batz S, Fluhr JW, Lademann J, Ulrich M. Assessment of a scoring system for basal cell carcinoma with multi-beam optical coherence tomography. J Eur Acad Dermatol Venereol 2015; 29: 1562-1569.

13. Coleman AJ, Richardson TJ, Orchard G, Uddin A, Choi MJ, Lacy KE. Histological correlates of optical coherence tomography in non-melanoma skin cancer. Skin Res Technol 2013; 19: 10-19.

14. Sattler E, Kastle R, Welzel J. Optical coherence tomography in dermatology. J Biomed Opt 2013; 18: 061224.

15. DeLong ER, DeLong DM, Clarke-Pearson DL. Comparing the areas under two or more correlated receiver operating characteristic curves: a nonparametric approach. Biometrics 1988; 44: 837-845.

16. van der Geer S, Frunt M, Romero HL, Dellaert NP, JansenVullers $\mathrm{MH}$, Demeyere TB, et al. One-stop-shop treatment for basal cell carcinoma, part of a new disease management strategy. Eur Acad Dermatol Venereol 2012; 26: 1154-1157.

17. Kadouch DJ, Wolkerstorfer A, Elshot $Y$, Zupan-Kajcovski B, Crijns MB, Starink MV, et al. Treatment of basal cell carcinoma using a one-stop-shop with reflectance confocal microscopy: study design and protocol of a randomized controlled multicenter trial. JMIR Res Protoc 2015; 4: e109.

18. Themstrup L, De Carvalho N, Nielsen SM, Olsen J, Ciardo S, Schuh $S$, et al. In vivo differentiation of common basal cell carcinoma subtypes by microvascular and structural imaging using dynamic optical coherence tomography. Exp Dermatol 2018; 27: 156-165.

19. Gambichler T, Orlikov A, Vasa R, Moussa G, Hoffmann K, Stucker $M$, et al. In vivo optical coherence tomography of basal cell carcinoma. J Dermatol Sci 2007; 45: 167-173. 